\title{
Synthesis and Antimicrobial Activities of some Quaternary Morpholinium Chlorides
}

\author{
BOGUMIŁ BRYCKI, ${ }^{1}$ ZOFIA DEGA-SZAFRAN ${ }^{1 *}$ and ILONA MIRSKA ${ }^{2}$ \\ ${ }^{1}$ Faculty of Chemistry, Adam Mickiewicz University, Poznań, Poland \\ ${ }^{2}$ Department of Pharmaceutical Bacteriology, University of Medical Sciences, Poznań, Poland
}

Received 10 April 2009, revised 15 January 2010, accepted 20 January 2010

\begin{abstract}
The synthesis and antimicrobial activity of 31 morpholinium chlorides, divided into five series depending on the substituents attached to the nitrogen atom, N-carboxyalkyl-morpholinium chlorides (1a-e), N-carbalkoxymethyl-N-methyl-morpholinium chlorides (2a-f), Ncarbethoxymethyl-N-alkyl-morpholinium chlorides (3a-g), N-carbalkoxymethyl-N-dodecyl-morpholinium chlorides (4a-f) and N-carboxymethyl-N-alkyl-morpholinium chlorides $(5 \mathrm{a}-\mathrm{g})$ is reported. The compounds investigated were tested for antimicrobial activity against Staphylococcus aureus, Escherichia coli, Proteus vulgaris, Pseudomonas aeruginosa, Candida albicans and Trichophyton menthagrophytes. The most active are compounds with a long $\mathrm{N}$-alkyl group and with the substituent $\mathrm{CH}_{2} \mathrm{COOC}_{\mathrm{n}} \mathrm{H}_{2 \mathrm{n}-1}(\mathrm{n}=8-16)$.
\end{abstract}

K e y w ords: antimicrobial activity, quaternary morpholinium chlorides

\section{Introduction}

Quaternary ammonium compounds have surfaceactive, detergent and antimicrobial properties (Fredell, 1994; Domingo, 1996). The largest area of application of quaternary ammonium salts is sanitation and disinfection. Cationic surfactants show membrane-disruptive properties, rapid antimicrobial activity and activity against a broad range of bacteria and fungi (Manivannan, 2008; Paulus, 2005; Fraise et al., 2004; Block, 2001). From among the large number of quaternary ammonium salts investigated, of particular interest are those in which the amino group is involved in the alicyclic ring, such as morpholinium, piperidinium or piperazinium. Their N,N-dialkyl derivatives display germicidal activity which is the greatest in the compounds containing long alkyl chains with from 12 to 16 carbon atoms. Their activity has been related to the structure, type of substituents and anions (Shelton et al., 1946). Recently, we have synthesized N-carboxymethyl-Nalkyl-piperidinium chlorides and $\mathrm{N}$-carbalkoxymethyl4-hydroxy-N-methyl-piperidinium chlorides, and tested them against some bacteria, yeast and fungi (Woźniak et al., 2004; Dega-Szafran et al., 2007). The promising pharmacological properties of this type of salts have prompted the synthesis of a series of morpholinium salts with N-alkyl, N-carboxyalkyl and N-carbalkoxymethyl groups attached to the nitrogen atom, of alkyl groups in a range of $\mathrm{C}_{1}-\mathrm{C}_{16}$ carbon atoms (Table I), to obtain more information on the effects of the structure and the alkyl chain on their biocidal activities against bacteria: Staphylococcus aureus, Escherichia coli, Proteus vulgaris, Pseudomonas aeruginosa and fungi: Candida albicans and Trichophyton menthagrophytes.

\section{Experimental}

\section{Materials and Methods}

Materials. N-Carboxyalkyl-morpholinium chlorides (1) were synthesized as described elsewhere (Dega-Szafran et al., 2001). N-carbalkoxymethyl-Nalkyl-morpholinum chlorides (2-4) were prepared by mixing equivalent amounts of the relevant $\mathrm{N}$-alkylmorpholine with the appropriate chloroacetic esters at room temperature. Crude product was washed with diethyl ether and recrystallized from acetonitrile. $\mathrm{N}$-Carboxymethyl-N-alkyl-morpholinium chlorides (5) were obtained from the corresponding esters (3) after reflux with $15 \% \mathrm{HCl}$ for 6 hours; removal of excessive water yielded products which were recrystallized

\footnotetext{
* Corresponding author: Z. Dega-Szafran, Faculty of Chemistry, Adam Mickiewicz University, ul. Grunwaldzka 6, 60-780 Poznań, Poland; phone: (+48) 61 8291216; fax: (+48) 61 8291505; e-mail: degasz@amu.edu.pl
} 
Table I

Melting points and selected ${ }^{1} \mathrm{H}$ and ${ }^{13} \mathrm{C}$ NMR chemical shifts in $\mathrm{CDCl}_{3}(\mathrm{ppm})$ for investigated morpholinium chlorides

\begin{tabular}{|c|c|c|c|c|c|c|}
\hline Compounds & No & $\mathrm{n}, \mathrm{R}_{1}, \mathrm{R}_{2}$ & M.p. $\left({ }^{\circ} \mathrm{C}\right)$ & $\delta{ }^{1} \mathrm{H} \mathrm{N}^{+} \mathrm{CH}_{2}$ & $\delta{ }^{13} \mathrm{C} \mathrm{N}^{+} \mathrm{CH}_{2}$ & $\delta{ }^{13} \mathrm{C} \mathrm{C}=\mathrm{O}$ \\
\hline \multirow{5}{*}{$\sim_{+}^{\mathrm{H}} \mathrm{Cl}^{-}-\left(\mathrm{CH}_{2}\right)_{n}-\mathrm{COOH}$} & $1 \mathrm{a}$ & 1 & $160-161^{\text {a }}$ & $3.88^{\mathrm{b}}$ & $57.24^{\mathrm{b}}$ & 168.43 \\
\hline & $1 \mathrm{~b}$ & 2 & $205-207^{\text {a }}$ & $3.31^{\mathrm{b}}$ & $53.34^{\mathrm{b}}$ & 174.45 \\
\hline & $1 \mathrm{c}$ & 3 & $183-184^{\text {a }}$ & $3.06^{\mathrm{b}}$ & $57.08^{\mathrm{b}}$ & 177.46 \\
\hline & $1 \mathrm{~d}$ & 4 & $156-157^{\text {a }}$ & $2.98^{b}$ & $57.57^{\mathrm{b}}$ & 177.33 \\
\hline & $1 \mathrm{e}$ & 5 & $179-182^{\text {a }}$ & $2.98^{\mathrm{b}}$ & $57.80^{\mathrm{b}}$ & 179.48 \\
\hline \multirow{6}{*}{$\overbrace{\mathrm{Cl}^{-}}^{\mathrm{CH}}$} & $2 \mathrm{a}$ & $\mathrm{C}_{8} \mathrm{H}_{17}$ & $125-128$ & 5.31 & 61.72 & 164.69 \\
\hline & $2 \mathrm{~b}$ & $\mathrm{C}_{10} \mathrm{H}_{21}$ & $131-133^{\mathrm{c}}$ & 5.34 & 61.70 & 164.75 \\
\hline & $2 \mathrm{c}$ & $\mathrm{C}_{11} \mathrm{H}_{23}$ & $94-96$ & 5.34 & 61.73 & 164.75 \\
\hline & $2 \mathrm{~d}$ & $\mathrm{C}_{12} \mathrm{H}_{25}$ & $108-110^{\mathrm{d}}$ & 5.33 & 61.79 & 164.73 \\
\hline & $2 \mathrm{e}$ & $\mathrm{C}_{15} \mathrm{H}_{31}$ & $107-110$ & 5.27 & 61.79 & 164.73 \\
\hline & $2 \mathrm{f}$ & $\mathrm{C}_{16} \mathrm{H}_{33}$ & $105-106^{\mathrm{e}, \mathrm{f}}$ & 5.33 & 61.75 & 164.77 \\
\hline \multirow{7}{*}{$\overbrace{\mathrm{Cl}^{-}}^{+\mathrm{R}_{2}}+\mathrm{CH}_{2}-\mathrm{COOC}_{2} \mathrm{H}_{5}$} & $3 a$ & $\mathrm{CH}_{3}$ & $172-174^{\mathrm{g}}$ & $5.13^{\mathrm{b}}$ & $60.25^{\mathrm{b}}$ & 164.61 \\
\hline & $3 b$ & $\mathrm{C}_{2} \mathrm{H}_{5}$ & $145-148$ & 5.13 & 60.25 & 164.81 \\
\hline & $3 \mathrm{c}$ & $\mathrm{C}_{3} \mathrm{H}_{7}$ & $150-151$ & 5.14 & 61.38 & 164.62 \\
\hline & $3 d$ & $\mathrm{C}_{4} \mathrm{H}_{9}$ & $63-64^{\mathrm{f}}$ & 5.04 & 59.97 & 164.65 \\
\hline & $3 \mathrm{e}$ & $\mathrm{C}_{8} \mathrm{H}_{17}$ & $132-135$ & 5.16 & 60.29 & 164.67 \\
\hline & $3 \mathrm{f}$ & $\mathrm{C}_{10} \mathrm{H}_{21}$ & $126-127$ & 5.16 & 60.28 & 164.61 \\
\hline & $3 \mathrm{~g}$ & $\mathrm{C}_{12} \mathrm{H}_{25}$ & $124-126$ & 5.13 & 60.52 & 164.61 \\
\hline \multirow{6}{*}{$\overbrace{\mathrm{Cl}^{-}}^{\mathrm{C}_{12} \mathrm{H}_{25}}$} & $4 a$ & $\mathrm{C}_{8} \mathrm{H}_{17}$ & $158-162^{\mathrm{f}}$ & 5.13 & 60.38 & 164.75 \\
\hline & $4 \mathrm{~b}$ & $\mathrm{C}_{10} \mathrm{H}_{21}$ & $130-132^{f}$ & 5.11 & 61.93 & 164.70 \\
\hline & $4 \mathrm{c}$ & $\mathrm{C}_{11} \mathrm{H}_{23}$ & $144-148^{\mathrm{f}}$ & 5.10 & 60.43 & 164.69 \\
\hline & $4 d$ & $\mathrm{C}_{12} \mathrm{H}_{25}$ & $82-84^{f}$ & 5.14 & 60.43 & 164.72 \\
\hline & $4 \mathrm{e}$ & $\mathrm{C}_{15} \mathrm{H}_{31}$ & $155-159^{\mathrm{f}}$ & 5.14 & 60.43 & 164.91 \\
\hline & $4 \mathrm{f}$ & $\mathrm{C}_{16} \mathrm{H}_{33}$ & $159-162^{f}$ & 5.11 & 60.42 & 164.70 \\
\hline \multirow{7}{*}{$\overbrace{\mathrm{Cl}^{-}}^{+\mathrm{R}_{2}} \mathrm{CH}_{2} \mathrm{COOH}$} & $5 \mathrm{a}$ & $\mathrm{CH}_{3}$ & $185-186^{\mathrm{h}}$ & $5.06^{b}$ & $63.01^{\mathrm{b}}$ & 167.37 \\
\hline & $5 \mathrm{~b}$ & $\mathrm{C}_{2} \mathrm{H}_{5}$ & $145-147$ & $4.16^{b}$ & $63.13^{b}$ & 167.36 \\
\hline & $5 \mathrm{c}$ & $\mathrm{C}_{3} \mathrm{H}_{7}$ & $234-237$ & $4.17^{b}$ & $62.85^{\mathrm{b}}$ & 167.64 \\
\hline & $5 \mathrm{~d}$ & $\mathrm{C}_{4} \mathrm{H}_{9}$ & $202-204$ & $4.14^{\mathrm{b}}$ & $61.43^{\mathrm{b}}$ & 167.72 \\
\hline & $5 e$ & $\mathrm{C}_{8} \mathrm{H}_{17}$ & $\mathrm{f}$ & $4.16^{b}$ & $61.47^{\mathrm{b}}$ & 168.29 \\
\hline & $5 \mathrm{f}$ & $\mathrm{C}_{10} \mathrm{H}_{21}$ & 98-99 & $4.14^{b}$ & $61.56^{\mathrm{b}}$ & 167.71 \\
\hline & $5 \mathrm{~g}$ & $\mathrm{C}_{12} \mathrm{H}_{25}$ & $90-93$ & $4.20^{\mathrm{b}}$ & $61.58^{\mathrm{b}}$ & 167.42 \\
\hline
\end{tabular}

${ }^{a}$ data from Dega-Szafran et al., 2001; ${ }^{\mathrm{b}}$ in $\mathrm{D}_{2} \mathrm{O} ;{ }^{\mathrm{c}}$ m.p. $157-158^{\circ} \mathrm{C}$ from Smith et al., 1951; ${ }^{\mathrm{d}}$ m.p. $113-114^{\circ} \mathrm{C}$ from Smith et al., 1951 ; ${ }^{\mathrm{e}}$ m.p. $119-120^{\circ} \mathrm{C}$ from Shelton et al., 1946; ${ }^{\mathrm{f}}$ hygroscopic compound; ${ }^{\mathrm{g}}$ data from Dega-Szafran et al., 2002a; ${ }^{\mathrm{h}}$ data from Dega-Szafran et al., $2002 \mathrm{~b}$.

from acetonitrile. The purity of the investigated chlorides was determined by elemental analysis and ${ }^{1} \mathrm{H}$ and ${ }^{13} \mathrm{C}$ NMR spectroscopy. Yields were $65-97 \%$ for chlorides $1,53-98 \%$ for $2,24-79 \%$ for $3,22-24 \%$ for $4,66-98 \%$ for 5 . The melting points and the ${ }^{1} \mathrm{H}$ and ${ }^{13} \mathrm{C}$ chemical shifts of the $\mathrm{N}^{+}-\mathrm{CH}_{2}-\mathrm{COO}$ fragments are listed in Table I.

Microbiological experiments. Standard strains were supplied by National Collection of Type Cultures (NCTC), London and American Type Culture Collection (ATCC). Minimum inhibitory concentration (MIC) was determined by the tube dilution method with inoculum to give $10^{5}$ microorganisms per $\mathrm{mL}$.
A series of chloride dilutions was prepared in MüllerHinton broth medium (bacteria) or in Sabouraud broth medium (fungi). The growth of microorganisms was determined visually and the lowest concentration of the chlorides that inhibited the multiplication of cells for $24 \mathrm{~h}$ at $37^{\circ} \mathrm{C}$ was taken as the MIC. MBCs of the tested chlorides were interpreted as follows: from each tube a sample was cultured on solid medium with inactivator $(2.5 \%$ lecithin, $5 \%$ lubrol $\mathrm{W}$ and $5 \%$ polysorbate 80 ) and results were read after incubation for $48 \mathrm{~h}$ at $37^{\circ} \mathrm{C}$ (bacteria) or at $28^{\circ} \mathrm{C}$ (fungi). The lowest concentration at which no colony formation was observed was defined as the MBC. 


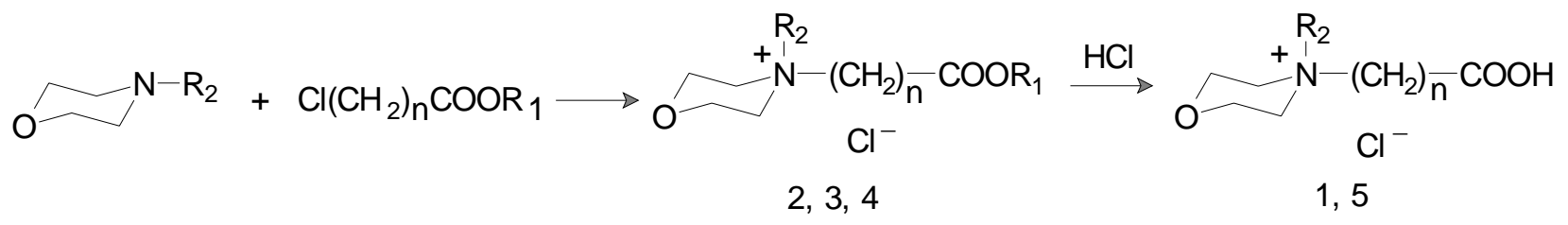

Fig 1. Synthesis of N,N-disubstituted morpholinium chlorides 1-5.

\section{Results and Discussion}

The morpholinium salts (1-5) were prepared according to the procedure outlined in Fig. 1 and can be divided into five series: (1) N-carboxyalkyl-morpholinium chlorides, (2) N-carbalkoxymethyl-N-methylmorpholinium chlorides, (3) N-carbethoxymethyl-Nalkyl-morpholinium chlorides, (4) N-carbalkoxymethyl-

Table II

Antimicrobial activity of morpholinium chlorides (1-5)

\begin{tabular}{|c|c|c|c|c|}
\hline \multirow{2}{*}{$\begin{array}{c}\text { Compound } \\
\text { No }\end{array}$} & \multicolumn{2}{|c|}{$\begin{array}{c}\text { Staphylococcus aureus } \\
\text { ATCC } 25923\end{array}$} & \multicolumn{2}{|c|}{$\begin{array}{c}\text { Pseudomonas aeruginosa } \\
\text { ATCC } 27853\end{array}$} \\
\hline & $\begin{array}{c}\mathrm{MIC} \\
\mu \mathrm{g} / \mathrm{ml}\end{array}$ & $\begin{array}{l}\mathrm{MBC} \\
\mu \mathrm{g} / \mathrm{ml}\end{array}$ & $\begin{array}{c}\mathrm{MIC} \\
\mu \mathrm{g} / \mathrm{ml}\end{array}$ & $\begin{array}{l}\mathrm{MBC} \\
\mu \mathrm{g} / \mathrm{ml}\end{array}$ \\
\hline $1 \mathrm{a}$ & 1250 & 2500 & 620 & 2500 \\
\hline $1 \mathrm{~b}$ & 5000 & 10000 & 5000 & 10000 \\
\hline $1 \mathrm{c}$ & 1250 & 5000 & 1250 & 2500 \\
\hline $1 \mathrm{~d}$ & 2500 & 5000 & 620 & 5000 \\
\hline $1 \mathrm{e}$ & 1250 & 2500 & 1250 & 5000 \\
\hline $2 a$ & 310 & 310 & 1250 & 2500 \\
\hline $2 b$ & 40 & 80 & 310 & 620 \\
\hline $2 c$ & 2.5 & 2.5 & 160 & 310 \\
\hline $2 d$ & 10 & 20 & 160 & 160 \\
\hline $2 \mathrm{e}$ & 5 & 10 & 620 & 620 \\
\hline $2 f$ & 10 & 20 & 620 & 1250 \\
\hline $3 a$ & $>10000$ & $>10000$ & $>10000$ & $>10000$ \\
\hline $3 b$ & $>10000$ & $>10000$ & $>10000$ & $>10000$ \\
\hline $3 \mathrm{c}$ & $>10000$ & $>10000$ & $>10000$ & $>10000$ \\
\hline $3 d$ & $>10000$ & $>10000$ & $>10000$ & $>10000$ \\
\hline $3 \mathrm{e}$ & 620 & 2500 & 1250 & 10000 \\
\hline $3 \mathrm{f}$ & 160 & 310 & 310 & 1250 \\
\hline $3 g$ & 5 & 10 & 160 & 620 \\
\hline $4 a$ & 0.62 & 0.62 & 20 & 40 \\
\hline $4 \mathrm{~b}$ & 5 & 5 & 160 & 310 \\
\hline $4 \mathrm{c}$ & 5 & 5 & 310 & 620 \\
\hline $4 d$ & 160 & 310 & 2500 & 5000 \\
\hline $4 \mathrm{e}$ & 310 & 310 & 1250 & 2500 \\
\hline $4 \mathrm{f}$ & 620 & 620 & 2500 & 2500 \\
\hline $5 a$ & 620 & 2500 & 620 & 2500 \\
\hline $5 \mathrm{~b}$ & 2500 & 5000 & 1250 & 2500 \\
\hline $5 c$ & 2500 & 5000 & 620 & 2500 \\
\hline $5 \mathrm{~d}$ & 1250 & 5000 & 1250 & 2500 \\
\hline $5 \mathrm{e}$ & 2500 & 10000 & 1250 & 5000 \\
\hline $5 \mathrm{f}$ & 310 & 620 & 620 & 1250 \\
\hline $5 \mathrm{~g}$ & 80 & 310 & 310 & 620 \\
\hline
\end{tabular}

$\mathrm{N}$-dodecyl-morpholinium chlorides and (5) N-carboxymethyl-N-alkyl-morpholinium chlorides (Table I).

The potential antimicrobial activity of compounds 1-5 was estimated in vitro by determining the MIC (minimal inhibitory concentration) and MBC (minimal bactericidal concentration) against cocci (Staphylococcus aureus), rods (Escherichia coli, Proteus vulgaris and Pseudomonas aeruginosa) and fungi (Candida albicans and Trichophyton menthagrophytes). The activity of the chlorides tested depends on the length of the alkyl chain attached to the quaternary nitrogen atom as well as in the ester group. Their antimicrobial activities against Staphylococcus aureus ATCC 25923 and Pseudomonas aeruginosa ATCC 27853 are listed in Table II. The compounds of series 2 and 4 were found to be very active, the most active being $4 \mathrm{a}$. The minimum bactericidal and fungicidal concentrations against Staphylococcus aureus NCTC 4163, Escherichia coli NCTC 8196, Proteus vulgaris NCTC 4635, Pseudomonas aeruginosa NCTC 6749, Candida albicans ATCC 10231 and Trichophyton menthagrophytes ATCC 9533 are listed in Table III. Based on decreasing activity against Staphylococcus aureus NCTC 4163, the order of the studied chlorides was $2 \mathrm{e}>2 \mathrm{~d} \sim 2 \mathrm{f} \sim 4 \mathrm{a}>$ $2 \mathrm{c} \sim 3 \mathrm{~g} \sim 4 \mathrm{~b} \sim 4 \mathrm{c}$; against Escherichia coli their order was as follows: $2 b \sim 2 \mathrm{e}>2 \mathrm{~d}>2 \mathrm{c} \sim 3 \mathrm{f} \sim 3 \mathrm{~g} \sim 4 \mathrm{a}$. Chlorides $2 \mathrm{~b}, 2 \mathrm{e}, 2 \mathrm{c}, 2 \mathrm{~d}, 3 \mathrm{f}, 3 \mathrm{~g}$ and $4 \mathrm{a}$ were active against Proteus vulgaris NCTC 4635, while only $2 \mathrm{c}, 2 \mathrm{~d}$ and 2e were active against Pseudomanas aeruginosa NCTC 6749. The activities of morpholinium chlorides against Candida albicans ATCC 10231 and Trichophyton menthagrophytes ATCC 9533 were very low, except for $2 \mathrm{e}, 2 \mathrm{~d}, 4 \mathrm{a}$ and $4 \mathrm{~b}$.

The quaternary ammonium compounds are primarily active against Gram-positive bacteria, with concentration as low as $5 \mu \mathrm{g} / \mathrm{ml}$ being lethal; higher concentration are lethal to Gram-negative bacteria, although Pseudomonas aeruginosa tends to be highly resistant (MIC $>100 \mu \mathrm{g} / \mathrm{ml}$ ) (Fraise et al., 2004). For example, the most known monoalkylammonium microbiocide, i.e. benzalkonium chloride, has a MIC value against Streptococcus agalactiae of $3.12, \mu \mathrm{g} / \mathrm{ml}$ (Mosca et al., 2006). On the other hand, didecyldimethyl ammonium chloride, the most frequently used active substance in biocidal preparations, shows minimum lethal concentration against Staphylococcus aureus at $32 \mu \mathrm{g} / \mathrm{ml}$ (Takasaki et al., 1994). The results obtained for 
Table III

Bactericidal and fungicidal activity $\left(\mathrm{MBC}_{15}\right.$ in $\left.\mathrm{mg} / \mathrm{ml}\right)$ of morpholinium chlorides $1-5$

\begin{tabular}{|c|c|c|c|c|c|c|}
\hline $\begin{array}{l}\text { Compound } \\
\text { No }\end{array}$ & $\begin{array}{c}\text { Staphylococcus } \\
\text { aureus } \\
\text { NCTC } 4163\end{array}$ & $\begin{array}{c}\text { Escherichia } \\
\text { coli } \\
\text { NCTC } 8196\end{array}$ & $\begin{array}{c}\text { Proteus } \\
\text { vulgaris } \\
\text { NCTC } 4635\end{array}$ & $\begin{array}{c}\text { Pseudomonas } \\
\text { aeruginosa } \\
\text { NCTC } 6749\end{array}$ & $\begin{array}{c}\text { Candida } \\
\text { albicans } \\
\text { ATCC } 10231\end{array}$ & $\begin{array}{c}\text { Trichophyton } \\
\text { menthagrophytes } \\
\text { ATCC } 9533\end{array}$ \\
\hline $1 \mathrm{a}$ & $>10000$ & $>10000$ & 5000 & 2000 & $>10000$ & $>10000$ \\
\hline $1 \mathrm{~b}$ & $>10000$ & $>10000$ & $>10000$ & $>10000$ & $>10000$ & $>10000$ \\
\hline $1 \mathrm{c}$ & $>10000$ & $>10000$ & $>10000$ & $>10000$ & $>10000$ & $>10000$ \\
\hline $1 \mathrm{~d}$ & $>10000$ & $>10000$ & $>10000$ & $>10000$ & $>10000$ & $>10000$ \\
\hline $1 \mathrm{e}$ & $>10000$ & $>10000$ & $>10000$ & $>10000$ & $>10000$ & $>10000$ \\
\hline $2 \mathrm{a}$ & 5000 & 2000 & 2000 & 5000 & $>10000$ & 10000 \\
\hline $2 b$ & 1000 & 50 & 100 & 2000 & $>10000$ & 500 \\
\hline $2 \mathrm{c}$ & 500 & 500 & 500 & 100 & $>10000$ & 2000 \\
\hline $2 d$ & 100 & 100 & 500 & 500 & 500 & 100 \\
\hline $2 \mathrm{e}$ & 50 & 50 & 100 & 500 & 100 & 1000 \\
\hline $2 f$ & 100 & 1000 & 1000 & 1000 & 100 & 1000 \\
\hline $3 a$ & $>10000$ & $>10000$ & $>10000$ & $>10000$ & $>10000$ & $>10000$ \\
\hline $3 b$ & $>10000$ & $>10000$ & $>10000$ & $>10000$ & $>10000$ & $>10000$ \\
\hline $3 c$ & $>10000$ & $>10000$ & $>10000$ & $>10000$ & $>10000$ & $>10000$ \\
\hline $3 \mathrm{~d}$ & $>10000$ & $>10000$ & $>10000$ & $>10000$ & $>10000$ & $>10000$ \\
\hline $3 \mathrm{e}$ & $>10000$ & 10000 & 10000 & 10000 & 10000 & 5000 \\
\hline $3 \mathrm{f}$ & 5000 & 500 & 500 & 1000 & 5000 & 1000 \\
\hline $3 \mathrm{~g}$ & 500 & 500 & 500 & 5000 & 5000 & 2000 \\
\hline $4 \mathrm{a}$ & 100 & 500 & 500 & 5000 & 100 & 100 \\
\hline $4 b$ & 500 & 1000 & 2000 & 5000 & 100 & 1000 \\
\hline $4 c$ & 500 & 1000 & 10000 & 10000 & $>10000$ & 1000 \\
\hline $4 d$ & 2000 & 10000 & 10000 & $>10000$ & $>10000$ & 1000 \\
\hline $4 \mathrm{e}$ & $>10000$ & 2000 & 10000 & $>10000$ & $>10000$ & 1000 \\
\hline $4 \mathrm{f}$ & 10000 & 1000 & 10000 & 10000 & $>10000$ & 1000 \\
\hline $5 \mathrm{a}$ & $>10000$ & $>10000$ & 5000 & 10000 & $>10000$ & $>10000$ \\
\hline $5 b$ & $>10000$ & $>10000$ & 5000 & 5000 & $>10000$ & $>10000$ \\
\hline $5 c$ & $>10000$ & $>10000$ & 5000 & 5000 & $>10000$ & $>10000$ \\
\hline $5 \mathrm{~d}$ & $>10000$ & $>10000$ & 5000 & 5000 & $>10000$ & $>10000$ \\
\hline $5 e$ & $>10000$ & 10000 & 5000 & 5000 & $>10000$ & $>10000$ \\
\hline $5 \mathrm{f}$ & $>10000$ & 5000 & 5000 & 2000 & $>10000$ & $>10000$ \\
\hline $5 \mathrm{~g}$ & 5000 & 5000 & 2000 & 1000 & $>10000$ & $>10000$ \\
\hline
\end{tabular}

quaternary morpholinium chlorides show that some of them, especially those with one or two long alkyl chains $\left(\mathrm{C}_{12}-\mathrm{C}_{16}\right)$, have biocidal activity comparable to the activity of benzalkonium chloride and didecyldimethylammonium chloride. It is also well known that the activity of microbiocides can be enhanced by the addition of some other compounds, like sequestrants and arylalkylalkohols as well by an increase of $\mathrm{pH}$ (Fraise et al., 2004). Market biocidal preparations contain not only active substances, one or more, but also contain some other chemicals to gain better application properties as well to avoid an increase in bacterial resistance. The low MIC and MBC values for three of the studied compounds, i.e. $4 \mathrm{a}, 2 \mathrm{c}$ and $2 \mathrm{f}$, and the stability of these compounds in water solu- tions in the presence of sequestrants, clearly indicate that the above compounds can be exploited as active substances in biocidal preparations.

Conclusions. Thirty one morpholinium chlorides have been tested for antimicrobial activities against Staphylococcus aureus, Escherichia coli, Proteus vulgaris, Pseudomonas aeruginosa, Candida albicans and Trichophyton menthagrophytes. The best biocidal results have been obtained for N-carbundecyloxymethyl-N-methyl-morpholinium chlorides (2c) and $\mathrm{N}$-carboctyloxymethyl-N-dodecyl-morpholinium chlorides (4a) against Staphylococcus aureus. In general, the most active are compounds with a long $\mathrm{N}$-alkyl group and with $\mathrm{CH}_{2} \mathrm{COOC}_{\mathrm{n}} \mathrm{H}_{2 \mathrm{n}-1}(\mathrm{n}=8-16)$ substituent. 
Acknowledgement

The authors thank Mrs E. Kiersztan, M. Pawlak and M. Śmigielska for technical assistance.

\section{Literature}

Block S.S. (ed.) 2001. Disinfection, Sterilization, and Preservation, (ed.:), Lippincott Williams and Wilkins, $5^{\text {th }}$ Edition, Philadelphia USA.

Dega-Szafran Z., Gąszczyk I., Maciejewska D., Szafran M., Tykarska E. and Wawer I. 2001. ${ }^{13} \mathrm{C}$ CP MAS NMR, FTIR $\mathrm{X}$-Ray diffraction and PM3 studies of some N-(w-carboxyalkyl) morpholine hydrohalides. J. Mol. Struct. 560: 261-273.

Dega-Szafran Z., Antkowiak A., Grundwald-Wyspiańska M., Nowak E., Gdaniec M. and Kosturkiewicz Z. 2002a. Structure and hydrogen bonding in a 3:2 inclusion compound of N-methylmorpholine betaine hydrochloride with acetone. J. Mol. Struct. 605: 325-330.

Dega-Szafran Z., Antkowiak A., Grundwald-Wyspiańska M., Nowak E. and Kosturkiewicz Z. 2002b. Crystal structure of bis(N-methylmorpholine betaine) hydrochloride, FTIR and NMR studies of 1:1 and 2:1 complexes of N-methylmorpholine betaine with mineral acids. J. Mol. Struct. 605: 309-317.

Dega-Szafran Z., Dulewicz E. and Brycki B. 2007. Synthesis and characterization of 1-carbalkoxymethyl-4-hydroxy-1-methylpiperidinium chlorides. ARKIVOC, part vi: 90-102 (http:/www. Arkat-usa.org)

Domingo X. 1996. Betaines. In Lomax E.G. (ed) Amphoteric Surfactants, Marcel Dekker Inc., New York USA Vol. 59, pp. 76-190,
Fraise A.P., Lambert P.A. and Maillard J-Y. (eds). 2004. Russell, Hugo and Ayliffe's Principles and Practice of Disinfection, Preservation and Sterilization. Blackwell Publishing, ${ }^{\text {th }}$ Edition, Malden USA.

Fredell D.L. 1994. Biological Properties and Applications of Cationic Surfactants in Cationic Surfactants (Analytical and Biological Evaluation) Cross J. and Singer E.J. (eds), Marcel Dekker Inc., New York USA, Vol. 53, 31-60.

Manivannan G. (ed.) 2008. Disinfection and Decontamination; Principles, Applications and Related Issues, CRC Press Taylor \& Francis Group, Boca Raton USA.

Mosca A., Russo F. and Miragliotta G. 2006. In vitro antimicrobial activity of benzalkonium chloride against clinical isolates of Streptococcus agalactiae. 57: 566-568.

Paulus W. (ed.) 2005. Directory of Microbiocides for the Protection of Materials. A. Handbook, Springer, Dordrecht, The Netherlands. Shelton R.S., Van Campen M.G., Tilford C.H., Lang H.C., Nisonger L., Bandelin F.J. and Rubenkoenig H.L. 1946. Quaternary Ammonium Salts as Germicides. J. Am. Chem. Soc. 68 : 753-759.

Smith D.R., Maienthal M. and Eifert R.L. 1951. Quaternary Ammonium Salts of Heterocyclic Bases. J. Am. Chem. Soc. 73: 2964-2965.

Takasaki A., Hashida T., Fujiwara S., Kato K. and Nishihara T. 1994. Bactericidal action of a quaternary ammonium disinfectant, didecyldimethyl ammonium chloride, against Staphylococcus aureus. Jpn. J. Toxicol Environ. Health, 40: 344-350.

Woźniak E., Morzymas A., Czarny A., Kocięba B., RóżyckaRoszak B., Dega-Szafran Z., Dulewicz E. and Petryna M. 2004. Antimicrobial activity of N-alkoxycarbonylmethyl-N-alkylpiperidinium chlorides Z. Naturforsch. 59c: 782-786. 